\title{
Anti-Thrombotic Therapy after Trans-Catheter Aortic Valve Implantation: Time for Refinement
}

\author{
Efstathios lliodromitis Dimitrios Farmakis \\ Second Department of Cardiology, Attikon University Hospital, National and Kapodistrian University of \\ Athens Medical School, Athens, Greece
}

One-third of patients with symptomatic severe aortic stenosis (AS) used to be denied life-saving aortic valve replacement due to advanced age, frailty, or comorbidities that rendered them high-risk candidates for surgery [1]. This setting has dramatically changed since the introduction of trans-catheter aortic valve implantation (TAVI), initially indicated for symptomatic severe AS in inoperable and frail patients or those with a high surgical risk $[2,3]$. In the subsequent years, following the technical improvement of devices and valve delivery systems, the increase of implanters' experience and the documentation of the technique's safety and durability results, its use is being expanded to include intermediate-risk patients. As TAVI is becoming available to an increasing spectrum of AS patients, refinements of the procedure and supporting care become necessary.

Peri-procedural complications associated with TAVI include, apart from local anatomical or coronary artery adverse events, an increased risk of thromboembolism and bleeding. The risk of device thrombosis, leading to valve dysfunction and embolism, most importantly stroke, seems to persist after the peri-procedural period, being associated with aortic wall disruption, turbulent flow through the valve, and artificial surface exposure
[4]. In key randomized trials, including PARTNER A and $\mathrm{B}, \mathrm{CUSPT}$, and CHOICE, the reported incidence of stroke ranged between 4.7 and $7.2 \%$ at 30 days postTAVI and 6.0 and $10.0 \%$ at 1 year [4]. Similarly, real-life evidence from TAVI registries shows that stroke occurs in $2.0-6.1 \%$ of patients at 30 days post-implantation [4].

Thromboembolic prophylaxis is provided by antiplatelet therapy, but the optimal anti-platelet regimen remains hitherto under debate. In general, dual therapy with low-dose aspirin and the thienopyridine clopidogrel (DAPT) is recommended for a period ranging between 1 and 6 months post-implantation, followed by single therapy (SAPT) with low-dose aspirin indefinitely $[2,3,5]$. According to recent evidence on current practice in 45 TAVI centers across the world, DAPT is routinely prescribed for $82 \%$ of patients after TAVI, but usually withdrawn after the first 3 months in more than half of cases [6].

The efficacy and safety of DAPT over SAPT has been questioned by some studies, systemic reviews, and metaanalyses $[4,6,7]$. In one of these meta-analyses, based on 26 studies on a total of 11,781 patients, mortality, stroke, and bleeding rates after TAVI did not differ between DAPT and SAPT [6].

\section{KARGER}

(c) 2018 S. Karger AG, Basel

E-Mail karger@karger.com

www.karger.com/crd
Dimitrios Farmakis, MD, PhD, FESC

Second Department of Cardiology

Attikon University Hospital, 1 Rimini St

GR-12462 Athens (Greece)

E-Maildimitrios_farmakis@yahoo.com 
The issue of anti-platelet therapy in patients with TAVI becomes more complicated in the presence of comorbid conditions that require anti-coagulation, such as atrial fibrillation (AF) or other systemic hypercoagulable states. In fact, AF is expected as a frequent comorbidity in TAVI candidates, as both AS and AF increase significantly with age. There is no consensus on the optimal anti-thrombotic regimen in these cases; US and Canadian guidelines advise against triple therapy, promoting the combination of low-dose aspirin with an oral anti-coagulant, while European guidelines suggest treating these patients with oral anti-coagulants according to atrial fibrillation guidelines, stating that the need to add an anti-platelet agent remains to be determined $[2,3,5]$. According to the recent European guidelines for valvular heart disease, new oral anti-coagulants, although contraindicated in patients with mechanical aortic valve prosthesis, may be considered as an alternative to vitamin $\mathrm{K}$ antagonists after the third month of implantation in patients who have atrial fibrillation with a surgical or transcatheter aortic valve bioprosthesis [8]. In the USA, the FDA-approved labelling of anti-coagulants continues to contraindicate the use of non-vitamin K antagonists for any prosthetic heart valve.

In this issue of Cardiology, $\mathrm{Hu}$ et al. [9] shed some new light on the topic by reporting the results of their meta-analysis comparing DAPT and SAPT post-TAVI. The authors have included a total of 7 studies, 4 randomized controlled trials, and 3 observational studies, with a total number of 1,445 patients. Five of the trials concerned the balloon-expendable SAPIEN device, and the remaining 2 involved the self-expandable Core-Valve device. According to this meta-analysis, DAPT carried an at least 2.5-fold-higher risk of major and life-threatening bleeding at 1,6 , and 12 months after implantation, with no benefit over SAPT in terms of all-cause mortality, stroke, or myocardial infarction at the same time points.

Hu et al. [9] analyzed the effects of DAPT versus SAPT in patients with the first-generation, and thus more thrombogenic, devices, i.e., the SAPIEN and the Core-Valve. Technical advances in the next-generation valves, along with the increasing implantation experience, are expected to minimize thrombogenicity, so the efficacy and safety of anti-thrombotic agents has to be revisited.

On the other hand, the recent REAC-TAVI trial showed that platelet reactivity remained high at 3 months post-TAVI in $79 \%$ of patients receiving typical DAPT with clopidogrel and aspirin [10]. Ticagrelor, in contrast, was associated with $100 \%$ platelet inhibition at 3 months, with no additional risk of major bleeding at 1 and 3 months in this very study [10]. It should be stressed, however, that this was a small trial with only 65 patients and the use of ticagrelor post-TAVI remains off-label.

Overall, the existing evidence questions the benefits of DAPT over SAPT after TAVI, even though it is largely based on previous-generation and thus more thrombogenic devices. A reasonable question that then arises, also considering the results of REAC-TAVI, is which agent would be more suitable in terms of efficacy and safety if one considers SAPT post-TAVI.

The durable results of TAVI, the reduction of periand post-procedural complications, and the lower bleeding risk that come with the meticulous use of proper anti-thrombotic agents for a short period of time would soon allow the broadening of TAVI indications to include younger patients without severe comorbidities. Given the mostly observational nature of clinical studies, the relatively low number of patients, and the short duration of follow-up, larger and longer randomized trails are warranted to define the optimal antithrombotic regimen in currently used devices, and to clarify the potential role of newer anti-platelet and anticoagulant agents. Ongoing studies testing different anti-thrombotic regimens, such as ATLANTIS (NCT02664649), AUREA (NCT01642134), AVATAR (NCT02735902), ENVISAGE-TAVI (NCT02943785), GALILEO (NCT02556203), and POPular-TAVI (NCT02247128), are expected to provide more solid evidence in this fascinating and rapidly growing field.

\section{Disclosure Statement}

There were no conflicts of interest.

\section{Funding Sources}

There was no funding. 


\section{References}

1 Iung B, Cachier A, Baron G, Messika-Zeitoun D, Delahaye F, Tornos P, et al. Decision-making in elderly patients with severe aortic stenosis: why are so many denied surgery? Eur Heart J. 2005 Dec;26(24):2714-20.

2 Vahanian A, Alfieri O, Andreotti F, Antunes MJ, Barón-Esquivias G, Baumgartner $\mathrm{H}$, et al.; Joint Task Force on the Management of Valvular Heart Disease of the European Society of Cardiology (ESC); European Association for Cardio-Thoracic Surgery (EACTS). Guidelines on the management of valvular heart disease (version 2012). Eur Heart J. 2012 Oct;33(19):2451-96.
3 Nishimura RA, Otto CM, Bonow RO, Carabello BA, Erwin JP 3rd, Fleisher LA, et al. 2017 AHA/ACC Focused Update of the 2014 AHA/ACC Guideline for the management of patients with valvular heart disease: a report of the American College of Cardiology/ American Heart Association Task Force on Clinical Practice Guidelines. J Am Coll Cardiol. 2017 Jul;70(2):252-89.

4 Vavuranakis M, Kalogeras K, Kolokathis AM, Vrachatis D, Magkoutis N, Siasos G, et al. Antithrombotic therapy in TAVI. J Geriatr Cardiol. 2018 Jan;15(1):66-75.

5 Webb J, Rodés-Cabau J, Fremes S, Pibarot P, Ruel M, Ibrahim R, et al. Transcatheter aortic valve implantation: a Canadian Cardiovascular Society position statement. Can J Cardiol. 2012 Sep-Oct;28(5):520-8.

6 Ahmad Y, Demir O, Rajkumar C, Howard JP Shun-Shin M, Cook C, et al. Optimal antiplatelet strategy after transcatheter aortic valve implantation: a meta-analysis. Open Heart. 2018 Jan;5(1):e000748.
7 Turgeon RD, Barry AR. Single vs dual antiplatelet therapy following transcatheter aortic valve implantation: A Systematic Review. Clin Cardiol. 2015 Oct;38(10):629-34.

8 Baumgartner H, Falk V, Bax JJ, De Bonis M, Hamm C, Holm PJ, et al.; ESC Scientific Document Group. 2017 ESC/EACTS Guidelines for the management of valvular heart disease. Eur Heart J. 2017 Sep;38(36):2739-91.

9 Hu X, Yang FY, Zhang Y, Wang Y. Single versus dual antiplatelet after transcatheter aortic valve implantation: A systematic review and meta-analysis. Cardiology. DOI: 10.1159/ 000490307.

10 Jimenez-Diaz VA. Assessment of Platelet Reactivity after Transcatheter Aortic Valve Implantation: REAC TAVI trial. CRT 2018 (abstr). 